\title{
Study of the dispersion of natural gas issuing from compressor stations through silencers with upper cover
}

\author{
J. García ${ }^{a}, *$ E. Migoya ${ }^{a}$, J.A. Lana ${ }^{b}$, A. Crespo ${ }^{a}$ \\ "Laboratorio de Mecánica de Fluidos, Departamento de Ingeniería Energética y Fluidomecánica, Escuela Técnica Superior \\ Ingenieros Industriales (ETSII), Universidad Politécnica de Madrid (UPM), Spain \\ ${ }^{\mathrm{b}}$ Enagás, S.A., Direction of Engineering and Gas Technology, Zaragoza, Spain
}

\begin{abstract}
The aim of the present study is the simulation of the dispersion of natural gas issuing from the silencer of compressor stations during vent operations. The objective is to analyze the dispersion of the gas emitted under different conditions of mass flow rate at the exit and ambient cross-flow velocity. We have considered a silencer with an upper cover to protect it from the rain and the fall of objects. The influence of the upper cover of the silencer on the dispersion of natural gas has also been studied, and non-dimensional approaches of the model have been proposed to simplify the problem. Seven different cases have been solved, using two models: a 3D model based on the commercial code FLUENT, and a simplified quasi-one-dimensional model. The results obtained in both cases have been compared, and the range of validity of the one-dimensional model in non-dimensional form has been discussed.
\end{abstract}

Keywords: Turbulent dispersion; Natural gas; Compressor station; CFD

\section{Introduction}

Natural gas compressor stations are installed in natural gas networks to compensate the pressure drop caused by the movement of the gas in the pipeline. In the operation of the compressor stations, it is necessary to accelerate the gas compressor by mean of an auxiliary system prior to start up the engine or gas turbine that drives the gas compressor (mechanical issues do not allow to start up the engine/gas turbine from zero rpm at the compressor). Traditionally, the easy and simple way to do this has been venting high pressure natural gas from the pipeline (normally more than $30 \mathrm{bar}$ ) through the compressor itself, where the expansion of the gas accelerates it to the required initial circular speed. This low pressure natural gas, about 6 bar, is venting to the atmosphere through a silencer that sometimes has an upper cover to protect it from the rain and fall of objects. This start up procedure is now been substituted by the utilization of start up electrical engines due to the envi- ronmental concerns caused by the emission of methane to the atmosphere.

Additionally, sometimes it is necessary to empty all the pressurized piping in the compressor station for maintenance works or any other cause. This is equally done through the silencer.

The analyzed silencer is shown in Fig. 1. The natural gas exits through two concentric circular rings; the diameter of the largest ring is $1.57 \mathrm{~m}$ approximately, and that of the inner one is $1.18 \mathrm{~m}$. The passage of the gas is hindered by the upper cover, which has the shape of an inverted cone, in such a way that the gas leaves the silencer with a radial component of velocity.

This kind of silencer with the upper cover has been utilized safely in compressor station for more than 20 years. The aim of the silencer is to reduce the noise in vent operations to a level below the regulations requirements and acceptable to the neighbors to the facility. The silencer is located in the middle of an exclusion zone, inside the limits of the compressor station, in order to provide enough space to disperse the gas cloud below the flammability limit without causing any hazard to the surroundings. However, in very particular operation and wind conditions, gas has been detected near the ground outside the limit of the exclusion zone; although the gas concentration is well below the 


\begin{tabular}{|c|c|}
\hline \multicolumn{2}{|c|}{ Nomenclature } \\
\hline \multicolumn{2}{|c|}{$A, B, C$ constants } \\
\hline$b$ & plume radius \\
\hline$d$ & parameter for the Gaussian profile \\
\hline$g$ & gravity \\
\hline$F_{0}$ & buoyancy flux \\
\hline$m_{0}$ & plume flow rate at the source \\
\hline$M_{0}$ & plume momentum at the source \\
\hline$r$ & radial coordinate \\
\hline$s$ & centre line \\
\hline$u$ & velocity \\
\hline$U$ & ambient air velocity \\
\hline$x$ & streamwise coordinate \\
\hline$y$ & transversal coordinate \\
\hline$Y$ & mass fraction \\
\hline$z$ & vertical coordinate \\
\hline \multicolumn{2}{|c|}{ Greek letters } \\
\hline \multicolumn{2}{|c|}{$\alpha(0.12), \beta(0.5), \varepsilon(0.125)$ entrainment coefficients } \\
\hline$\theta$ & $\begin{array}{l}\text { angle between the horizontal and plume centre } \\
\text { lines }\end{array}$ \\
\hline$\Lambda$ & non-dimensional momentum flux, Eq. (9’”) \\
\hline$\mu, \mu_{1}$ & $\begin{array}{l}\text { non-dimensional mass flux at origin, Eqs. (14) and } \\
\left(8^{\prime \prime \prime \prime)}\right.\end{array}$ \\
\hline$\rho$ & density \\
\hline$\varphi$ & scalar variable \\
\hline \multicolumn{2}{|c|}{ Subscripts } \\
\hline 0 & source of the plume $(s=0)$ \\
\hline 1 & $\begin{array}{l}\text { non-dimensional value for cases without upper } \\
\text { cover }\end{array}$ \\
\hline 2 & $\begin{array}{l}\text { non-dimensional value made for cases with upper } \\
\text { cover }\end{array}$ \\
\hline$a$ & ambient air \\
\hline$c$ & centre line \\
\hline$N$ & natural gas \\
\hline$<>$ & average value in the plume \\
\hline
\end{tabular}

flammability limit $(<1$ vol.\%), it is high enough to produce malfunctions of the gas turbine driving the compressors, if mixed with the combustion air of the turbine.

The generally accepted lower flammability limit (LFL) of the natural gas is the one of methane (main component, $>90 \mathrm{vol} . \%$ normally, of natural gas), which is set at a $5 \mathrm{vol} . \%$ in the literature related to industrial safety, see Lees' and Santamaría and Braña Recently, experiments have been reported in the literature to determine the flammability limits of the mixture of natural gas and air They found that the flammability region is from $5.0 \%$ to $15.6 \%$ of natural gas by volume at ambient conditions, which is comparable with that of pure methane in air. For standardization and certification purposes, the official LFL for methane is established at $4.4 \mathrm{vol} . \%$,
The objective of the present study is to analyze the dispersion of the gas emitted under different conditions of mass flow rate at the exit and of ambient cross-flow velocity, assuming steady state conditions. Seven different cases are solved, using two models: a three-dimensional model using the commercial code FLUENT, and a quasi-one-dimensional model, based on the work of Castro et al. and Servert The results obtained in both cases are compared, both in dimensional and non-dimensional form, and the range of validity of the two models is discussed. Special attention is paid to the influence of the presence of the upper cover of the silencer on the dispersion of natural gas. The behavior of the flow near the upper cover is simulated exactly with the 3D model and is implemented in the one-dimensional model by means of an artificial enlargement of the exit area.

1D models have been frequently used before to calculate the plume dispersion characteristics, because they were the only way to obtain results of engineering interest in reasonable computing times; but nowadays CFD codes are relatively quick to run and provide more accurate answers and can handle more complex flow and boundary conditions. However, the computing times of 3D models are still much larger than those of $1 \mathrm{D}$ models, and besides the times needed to run and reduce the data from a 1D model are also much shorter. In spite of the higher fidelity answer obtained with CFD calculations, the additional time requirement may not always be fully justified. The $1 \mathrm{D}$ models can provide quick answers for parametric studies, and later the obtained results can be confirmed with the 3D calculations. Because of this we have introduced in this paper a comparison of both $1 \mathrm{D}$ and $3 \mathrm{D}$ results, and suggested boundary condition for the releases with an upper cover, so that they can be studied with the 1D model. Besides, algebraic correlations giving plume trajectories and gas concentrations are also given in the paper for situations both with and without upper lid; these correlations are more easily deduced and justified with the 1D model equations. The parametric dependence of the results is also made more obvious by the non-dimensional analyses that have been introduced for cases both with and without upper cover, and can also be more easily deduced and justified with the 1D model equations. Another reason to use 1D models is that this can be used by any engineer with knowledge on gas dispersion phenomena while 3D model requires a specialist with a high degree of skills on CFD, both to create the grid and initial contour condition and to interpret the data, besides the fact that the software for CFD models is quite more complicated to use than the one of a $1 \mathrm{D}$ model.

The dispersion of plumes has been studied previously by many authors, analytical plume rise models can be found in Briggs and Ooms and Mahieu that, in order to evaluate plume trajectories, relate the plume mass and momentum fluxes to the forces acting on a plume and the entrainment rate of external fluid. A good review on turbulent jets and plumes was made Contini and Robins and MacDonald et al. investigated the factors that modify both plume trajectories and plumes shapes. They concluded that they are due to two different causes: the modification of the entrainment rate due to changes in speed relative to the cross-flow and to changes in plume shape during the mixing phase, and the velocity induced by the counter 

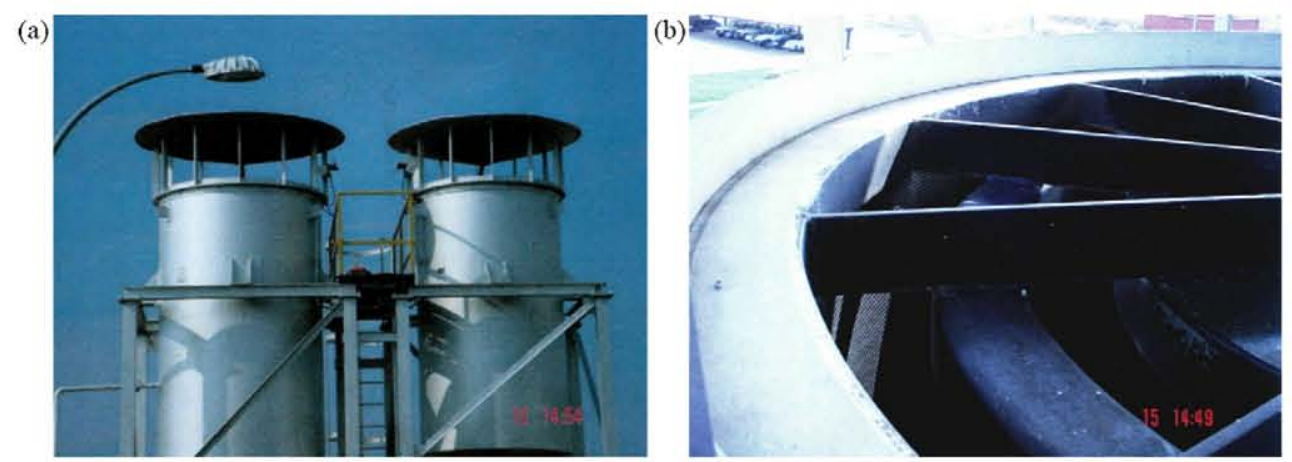

Fig. 1. (a) Side view of the silencers with the upper cover. (b) Detailed view of the exit.

rotating vortex pair. Seifert and Shemer showed that using non-circular nozzles at various orientations with respect to the cross stream can alter the jet penetration into the free stream; they found that when an elongated exit cross section is oriented along the wind direction, the chimney effective height is increased as compared to a circular exit cross section. In this article, we will focus on the influence of upper lid in the diffusion of the plume.

\section{One-dimensional model}

The model uses a quasi-one-dimensional treatment (see Fig. 2), assuming self-similar profiles for fluid magnitudes in planes perpendicular to the centre line of the plume, as described in Castro et al. for natural gas dispersion and in Servert et al.

for jet fires. In planes normal to the middle line, the perturbations over the ambient values of all dependent variables are largest at the centre line itself and decay and tend to zero for large enough values of the radial distance to the middle line. The profiles of fluid magnitudes are assumed to be Gaussian in sections normal to the plume trajectory:

$\varphi-\varphi_{\mathrm{a}}=\left(\varphi_{\mathrm{c}}-\varphi_{\mathrm{a}}\right) \exp \left(-\frac{2 r^{2}}{b^{2}}\right)$

where the variable $\varphi$ is: any of the velocity components or the gas mass fraction. Subscript $\mathrm{c}$ in $\varphi_{\mathrm{c}}$, means the value at the centre of the plume, and subscript a in $\varphi_{\mathrm{a}}$ the ambient external value; $r$ is the distance to the centre line and $b$ the equivalent top-hat radius. As shown in Servert et al. the Gaussian distributions

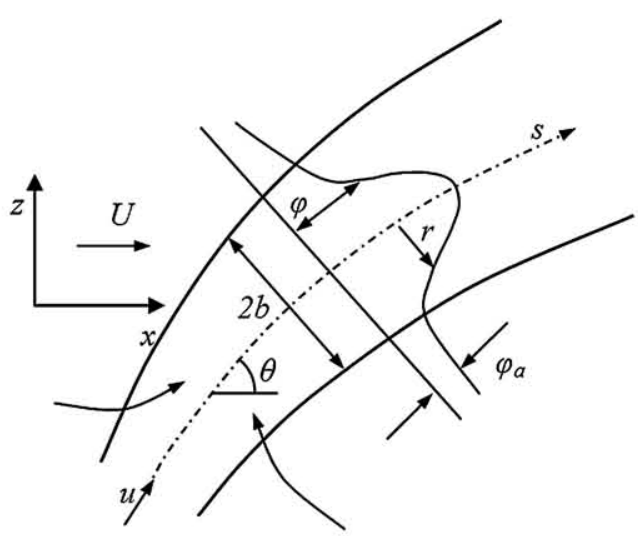

Fig. 2. Schematic showing the main parameters of the one-dimensional model. can be related to a top-hat profile (Fig. 3), and the following relationship between the top-hat values of the variables and the centre values of the Gaussian distribution is assumed:

$\left(\varphi_{\mathrm{c}}-\varphi_{\mathrm{a}}\right)=2\left(\langle\varphi\rangle-\varphi_{\mathrm{a}}\right)$

where $\langle\varphi\rangle$, is the averaged value of the variable $\varphi$ in a plane normal to the centre line. In the following the equations to calculate $\langle\varphi\rangle$ and $b$ will be given, and the brackets of $\langle\varphi\rangle$ will be omitted. For the average value of the velocity modulus the symbol $u$ will be used; the velocity components will be obtained from the plume inclination respect to the horizontal, $\theta$, so that $v_{x}=u \cos \theta, v_{z}=u \sin \theta$; the other velocity component is zero because the average velocity vector is supposed to be in a vertical plane that contains the exit axis. The average density will be denoted by $\rho$. The Boussinesq approximation will be used so that the density perturbations will be small compared to the ambient density: $\left|\rho-\rho_{\mathrm{a}}\right| \ll \rho_{\mathrm{a}}$. This assumption is not valid very near the exit, and will not be used in the $3 \mathrm{D}$ model. All the density variations are assumed to be due to changes in concentration, so that the mass fraction of natural gas will be given by:

$Y_{\mathrm{N}}=\frac{\rho_{\mathrm{a}}-\rho}{\rho_{\mathrm{a}}-\rho_{\mathrm{N}}}$,

where $\rho_{\mathrm{N}}$ is the density of the natural gas. Obviously, the ambient value of the gas concentration is zero: $Y_{\mathrm{Na}}=0$. For the air an average density of $\rho_{\mathrm{a}}=1.2 \mathrm{~kg} / \mathrm{m}^{3}$ has been assumed, whereas for the natural gas an average density of $\rho_{\mathrm{N}}=0.847 \mathrm{~kg} / \mathrm{m}^{3}$ has been considered.

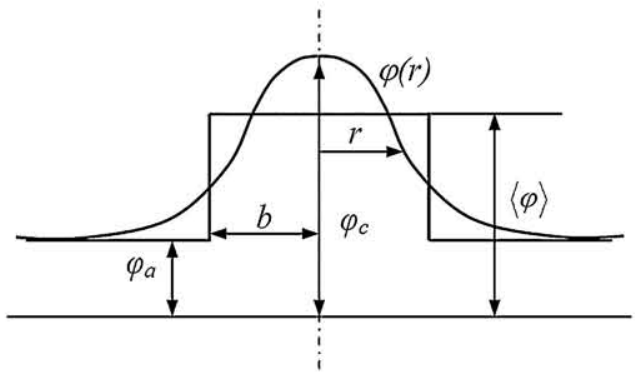

Fig. 3. Main parameters of the Gaussian and top-hat profiles. 


\subsection{Governing equations and boundary conditions}

\subsubsection{Mass conservation}

$$
\begin{aligned}
\frac{\mathrm{d}\left(\pi b^{2} u\right)}{\mathrm{d} s}= & 2 \pi b \alpha|u-U \cos \theta|+2 \pi b \beta|U \sin \theta| \\
& +2 \pi b \varepsilon|U \cos \theta|
\end{aligned}
$$

where $u$ is the velocity of the plume, $b$, the radius of the plume, $s$, the coordinate along the centre line, $U$, the wind velocity, and $\theta$ is the angle between the centre line and the horizontal line (see Fig. 1). The parameters $\alpha, \beta$, and $\varepsilon$ are the entrainment coefficients. The first two terms in the right hand side represent entrainment due to the relative velocities, parallel and normal to the plume, respectively, see Escudier [14]; the following values have been used $\alpha=0.12, \beta=0.5$. The last term has been added to take into account the plume dispersion due to ambient turbulence, that occurs even when the plume is horizontal and the wind and plume velocities are equal. The value of the corresponding coefficient has been taken as $\varepsilon=0.125$, and has been estimated from the numerical results obtained with the $3 \mathrm{D}$ code described later: it is also in agreement with classical values suggested by Pasquill and Smith [15] for neutral atmospheric conditions.

The sensitivity of the model results to parameter $\alpha$ can be inferred from the dimensional analysis of Section 5. The sensitivity to $\beta$ is important for cases in which the plume trajectory is very vertical; variations of $\beta$ leading to relative variations in concentrations and trajectories of the same order of the variation of $\beta$. On the other hand, the sensitivity to $\varepsilon$ is important for cases in which the plume trajectory is very horizontal, for high winds and low mass flow rates.

\subsubsection{Momentum conservation along the plume \\ $\frac{\mathrm{d}\left(\pi b^{2} u^{2}\right)}{\mathrm{d} s}=U \cos \theta \frac{\mathrm{d}\left(\pi b^{2} u\right)}{\mathrm{d} s}+\pi b^{2} g \frac{\rho_{\mathrm{a}}-\rho}{\rho_{\mathrm{a}}} \sin \theta$}

where $g$ is the gravity. The term on the left hand side represents the net momentum flux between two consecutive sections, the first term on the right hand side represents the momentum flux inside the plume due to entrainment, and the other one is due to buoyancy.

\subsubsection{Momentum conservation in a direction normal to the plume}

$\pi b^{2} u^{2} \frac{\mathrm{d} \theta}{\mathrm{d} s}=-U \sin \theta \frac{\mathrm{d}\left(\pi b^{2} u\right)}{\mathrm{d} s}+\pi b^{2} g \frac{\rho_{\mathrm{a}}-\rho}{\rho_{\mathrm{a}}} \cos \theta$
The term on the left hand side represents a centrifugal force, and on the right hand side the first term represents the momentum flux inside the plume due to entrainment, and the other one is due to buoyancy.

Eqs. (5) and (6) can be combined, and the resulting equation can be integrated, yielding the following algebraic relation that can substitute either of these two equations:

$\pi b^{2} u(u \cos \theta-U)=\mathrm{constant}$

This equation expresses global conservation of horizontal momentum

\subsubsection{Conservation equations of mass of gas and of buoyancy}

The conservation of mass of natural gas can be expressed as:

$\frac{\mathrm{d}\left(\pi b^{2} u Y_{\mathrm{N}}\right)}{\mathrm{d} s}=0 \Rightarrow \pi b^{2} u Y_{\mathrm{N}}=\mathrm{constant}$

Multiplying both sides of this last equation by $g\left[\left(\rho_{\mathrm{a}}-\rho_{\mathrm{N}}\right) / \rho_{\mathrm{a}}\right]$, and on using Eq. (3), it is obtained that

$$
\pi b^{2} u g \frac{\rho_{\mathbf{a}}-\rho}{\rho_{\mathbf{a}}}=F_{0}
$$

This equation expresses that there is not variation of buoyancy flux, that is equal to its value at the exit, $F_{0}$.

\subsubsection{Boundary conditions}

The boundary conditions at the exit for the previous equations express that the mass and momentum fluxes, $G=\rho_{\mathbf{a}} m_{0}$ and $G^{2} / \rho_{\mathrm{N}} A_{\text {eff }}$, respectively, are known, and that the jet is vertical:

$s=0 \rightarrow \pi b^{2} u=m_{0}=\frac{G}{\rho_{\mathrm{a}}}$

$s=0 \rightarrow \pi b^{2} u^{2}=M_{0}=\frac{G^{2}}{\rho_{\mathrm{N}} \rho_{\mathrm{a}} A_{\mathrm{eff}}}$

$s=0 \rightarrow \theta=\frac{\pi}{2}$

where $G$ is given in Table 1 , and for the cases without cover, $A_{\text {eff }}=0.882 \mathrm{~m}^{2}$ is the area between two concentric circles of radius $0.785 \mathrm{~m}$ and $0.680 \mathrm{~m}$ for the exterior ring, and $0.590 \mathrm{~m}$ and $0.470 \mathrm{~m}$ for the interior one. For the cases with cover, it is assumed that the lid eliminates most of the initial momentum flux and that the initial area is very large, in particular it has been taken as one hundred times larger than its real value, $A_{\mathrm{eff}}=88.2 \mathrm{~m}^{2}$.

\begin{tabular}{|c|c|c|c|c|c|c|}
\hline Case number & Wind velocity $(\mathrm{m} / \mathrm{s})$ & Mass flow rate of natural gas $(\mathrm{kg} / \mathrm{s})$ & Upper cover & $\mu_{1}$ & $\mu_{2}$ & $\Lambda_{2}$ \\
\hline 1 & 2 & 70 & Yes & - & $2.14 \mathrm{E}-04$ & $1.00 \mathrm{E}-05$ \\
\hline 2 & 2 & 70 & No & $1.61 \mathrm{E}-02$ & $2.14 \mathrm{E}-04$ & $1.00 \mathrm{E}-02$ \\
\hline 3 & 5 & 70 & No & $1.61 \mathrm{E}-02$ & $2.09 \mathrm{E}-02$ & $3.91 \mathrm{E}-01$ \\
\hline 4 & 10 & 5 & Yes & - & $9.39 \mathrm{E}+00$ & $6.25 \mathrm{E}-03$ \\
\hline 5 & 10 & 5 & No & $2.26 \mathrm{E}-01$ & $9.39 \mathrm{E}+00$ & $6.25 \mathrm{E}+00$ \\
\hline 6 & 10 & 70 & Yes & - & $6.67 \mathrm{E}-01$ & $6.25 \mathrm{E}-03$ \\
\hline 7 & 10 & 70 & No & $1.61 \mathrm{E}-02$ & $6.67 \mathrm{E}-01$ & $6.25 \mathrm{E}+00$ \\
\hline
\end{tabular}

Table 1

Mass flow rates, wind velocity, geometry, and non-dimensional parameters $\mu_{1}, \mu_{2}, A_{2}$ corresponding to the simulated cases 


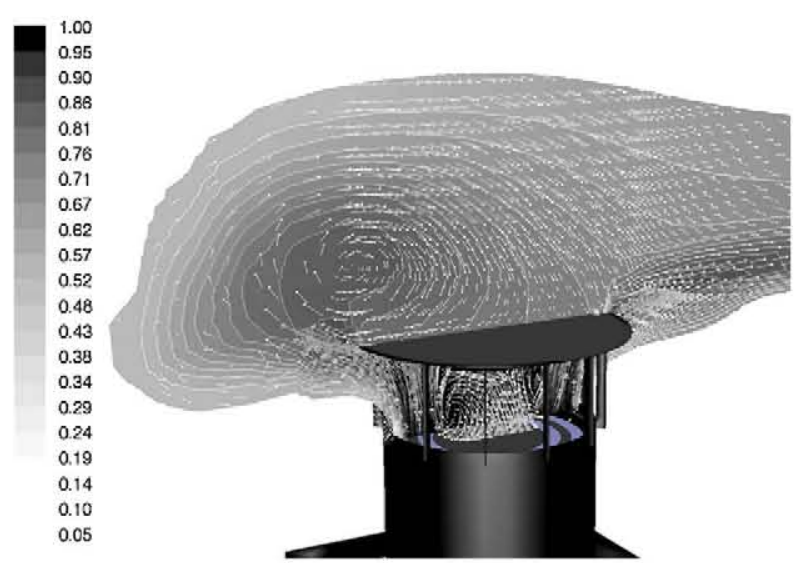

Fig. 4. Velocity vectors and mass concentration of natural gas at the symmetry plane for the case 6 (mass flow rate is $70 \mathrm{~kg} / \mathrm{s}$ and the wind velocity is $v=10 \mathrm{~m} / \mathrm{s}$, with upper cover).

The results obtained do not change if this area is increased further. In the dimensional analysis carried out later in this work, $M_{0}$ will be assumed to tend to zero. It will be shown, that for this condition to be satisfied the outgoing velocity (after the flow has been decelerated by the lid) should be much smaller than the wind velocity, $u_{0} \ll U$. In Fig. 4 it is shown how the effective area of the plume is highly increased by the upper cover, this

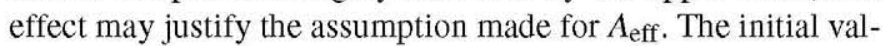
ues of $u=u_{0}$ and $b=b_{0}$, needed for numerical integration, are obtained from Eqs. (8) and (9).

\section{Three-dimensional model}

The three-dimensional simulations have been performed with the commercial code FLUENT that solves the equations discretized by means of the finite volume method. The equations of conservation of mass and momentum in the three directions have been solved. It is assumed that outside the silencer the Mach number is small enough, and the effects of compressibility induced by the pressure variations have not been taken into account. In addition, the temperature variations are not significant either and its influence has been neglected to calculate the density. Consequently, it has not been necessary to solve the equation of conservation of energy. The $k-\varepsilon$ turbulence model has been used for turbulence closure that implies the resolution of two additional equations, the equation of conservation of turbulent kinetic energy $(k)$ and the corresponding one to its

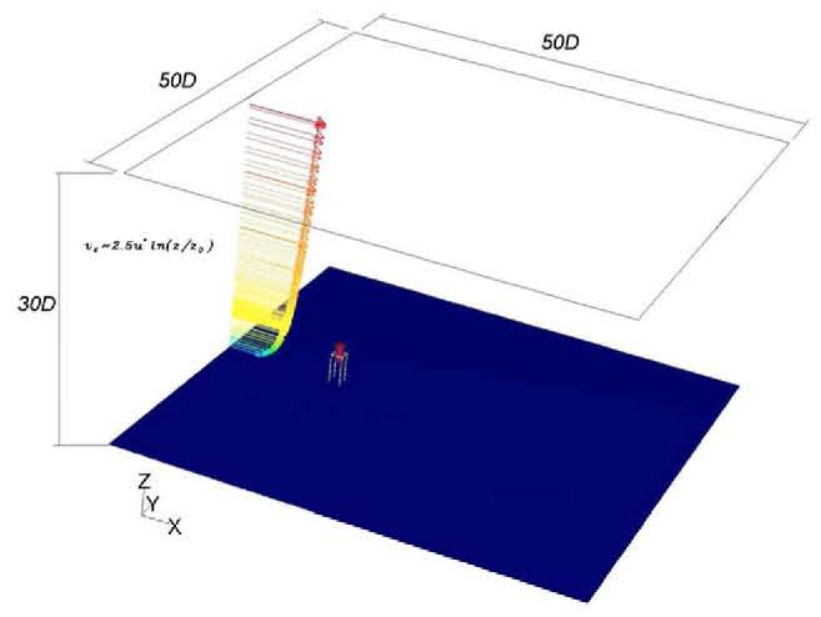

Fig. 6. Computational domain and reference system. The dimensions are referred to the diameter $D=2 \mathrm{~m}$ of the silencer.

dissipation $(\varepsilon)$. To calculate the natural gas concentration also the equation of conservation of that species had to be solved, considering the system as a binary mixture of air and natural gas. The coefficient of turbulent diffusion is also estimated by means of the $k-\varepsilon$ turbulence model.

The numerical resolution of the Navier-Stokes equations has been carried out using a second order upwind scheme for the convective terms and a central difference scheme for the diffusion terms. The coupling between equations through the pressure has been solved using the SIMPLE method.

The results provided by the FLUENT code have been validated against experiments by the authors in problems concerning hydrogen dispersion (Gallego et al. [16]) and concerning winds over complex topography (Migoya et al. [17]).

Regarding the mesh, with the exception of a small zone located under the upper cover that has been meshed with tetrahedral cells (Fig. 5b), in the rest of the computational domain a non-uniform structured hexahedral mesh has been used (Fig. 5a). The size of the used mesh was 2.1 million cells. This grid size has been chosen after performing a grid independence analysis of the solution. It has been checked that finer grids produce similar results. The domain dimensions, referred to the diameter of the silencer $D=2 \mathrm{~m}$, were $100 D \times 50 D \times 75 D$ in the $x, y$ and $z$ directions, respectively (see Fig. 6) to assure that the plume leaves the computational domain horizontally. The computations have been carried out in a two-processor server (SunFire 280R), the CPU times were about 1 week.
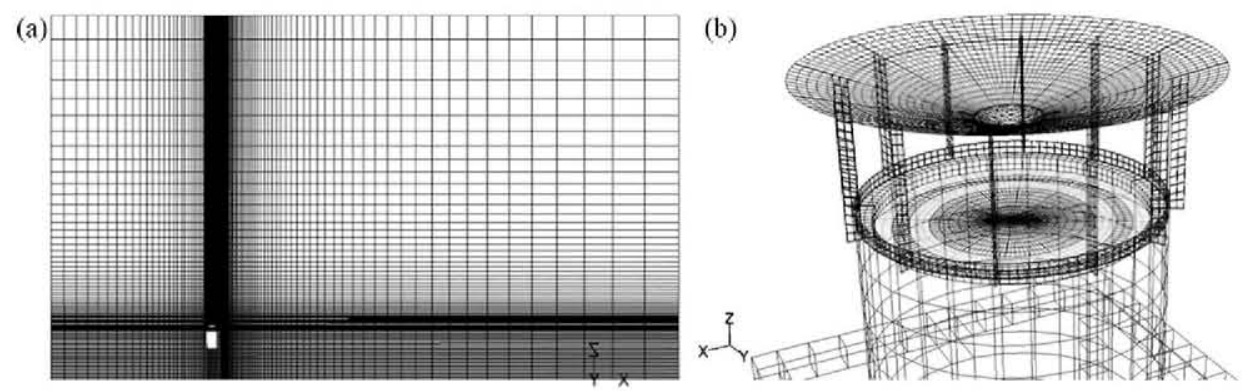

Fig. 5. (a) Computational grid at the symmetry plane. (b) Mesh at the outlet of the silencer. 

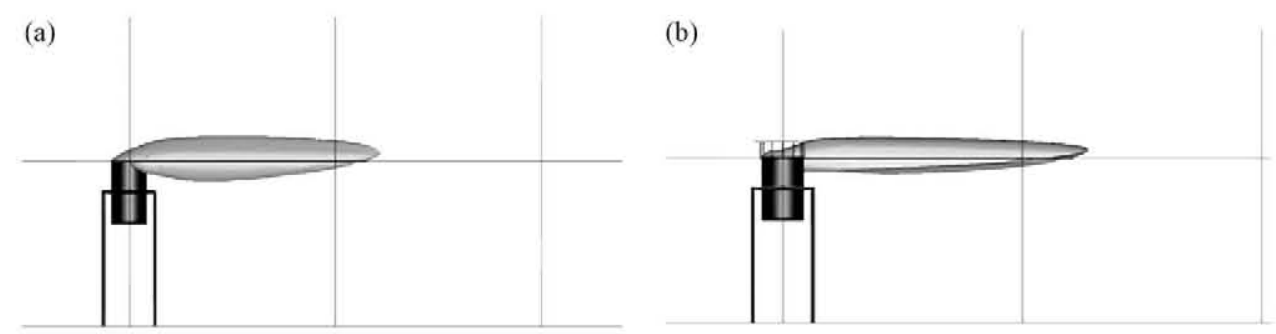

Fig. 7. Isosurface of concentration equal to $5 \%$. Mass flow rate is $5 \mathrm{~kg} / \mathrm{s}$ and the wind velocity is $10 \mathrm{~m} / \mathrm{s}$ : (a) without upper cover and (b) with upper cover. The distance between lines is $10 \mathrm{~m}$.

(1)

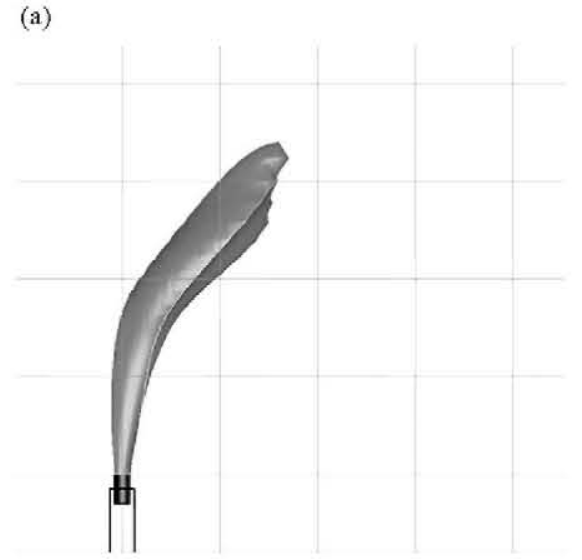

(b)

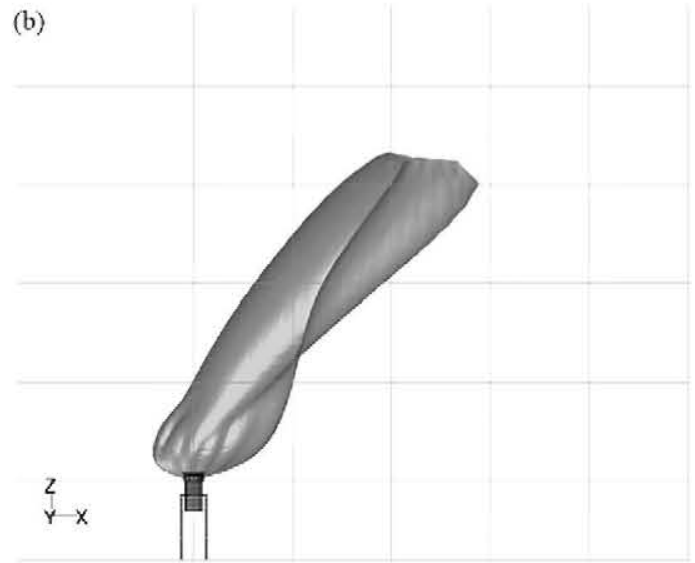

Fig. 8. Isosurface of concentration equal to $5 \%$. Mass flow rate is $70 \mathrm{~kg} / \mathrm{s}$ and the wind velocity is $2 \mathrm{~m} / \mathrm{s}$ : (a) without upper cover and (b) with upper cover. The distance between lines is $10 \mathrm{~m}$.

The boundary conditions imposed have been the following ones:

(a) Inlet conditions for the ambient wind. In the zone where the wind enters into the domain it has been assumed a logarithmic profile of speed, corresponding to the law of the wall (Fig. 6):

$$
v_{x}=2.5 u^{*} \ln \left(\frac{z}{z_{0}}\right)
$$

where $z$ is the distance to the ground, $z_{0}$ the roughness surface, in our case equal to $0.01 \mathrm{~m}$, and $u^{*}$ is the friction velocity, that has been adjusted to obtain at the height of the exit of the silencer, located at $8 \mathrm{~m}$ above the ground, the three different incident velocities considered in this work, $2 \mathrm{~m} / \mathrm{s}, 5 \mathrm{~m} / \mathrm{s}$, and $10 \mathrm{~m} / \mathrm{s}$.

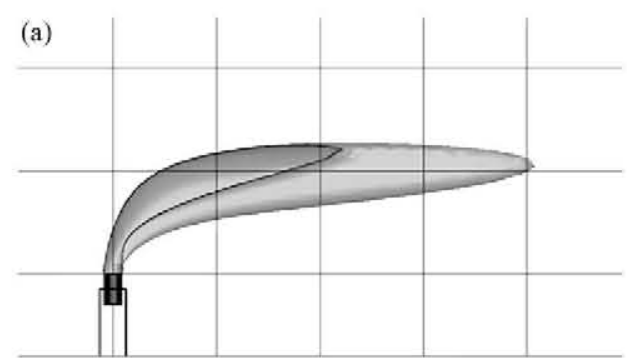

The inlet values of turbulent kinetic energy $k$ and its dissipation $\varepsilon$, are given by the following expressions:

$$
\begin{aligned}
& k=\frac{1}{\sqrt{C_{\mu}}} u^{* 2}=3,33 u^{* 2} \\
& \varepsilon=2.5 \frac{u^{* 3}}{z}
\end{aligned}
$$

where $C_{\mu}=0.09$. The values of the defined variables in Eqs. (11)-(13) are an exact solution of the $k-\varepsilon$ model, therefore the effect of natural gas dispersion can be considered as a non-linear perturbation of the inlet boundary condition for the ambient wind. For this reason, the values given in Eqs. (11)-(13) have been considered as the initialization values for the whole domain, this assumption accelerates the convergence of the numerical solution. (b)

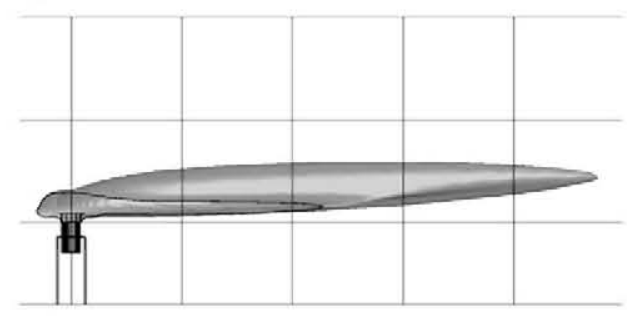

Fig. 9. Isosurface of concentration equal to $5 \%$. Mass flow rate is $70 \mathrm{~kg} / \mathrm{s}$ and the wind velocity is $10 \mathrm{~m} / \mathrm{s}$ : (a) without upper cover and (b) with upper cover. The distance between lines is $10 \mathrm{~m}$. 
(b) In the ground a null perturbation of the basic flow has been imposed.

(c) The exit of the silencer consists of two concentric circular rings, of dimensions indicated in the introduction. Two different mass flow rates have been considered $5 \mathrm{~kg} / \mathrm{s}$ and $70 \mathrm{~kg} / \mathrm{s}$. At the exit a uniform velocity has been assumed corresponding to each mass flow rate and the mass fraction of natural gas equal to one.

(d) In the remaining boundary surfaces the ambient pressure has been fixed.

\section{Results, discussion and comparison of the two models}

The dispersion of the natural gas has been calculated for seven different cases as it is shown in Table 1. In the table are indicated the wind velocity, mass flow rate of natural gas, the geometry (whether they have or not upper cover) and finally several nondimensional parameters that will be explained later.

\subsection{Plume description obtained from the $3 D$ model}

In Figs. 7-9 the isosurfaces corresponding to a mass concentration equal to $5 \%, Y_{\mathrm{N}}=0.05$, are presented. The isosurfaces are cut by the vertical symmetry plane and also include a view of the isocontour surface on the back. In both Figs. 7 and 8 a, that there is no bifurcation both views are undistinguishable. In these figures we can compare the effect of two different mass flow rates $(5 \mathrm{~kg} / \mathrm{s}$ and $70 \mathrm{~kg} / \mathrm{s})$, two different wind velocities $(2 \mathrm{~m} / \mathrm{s}$ and $10 \mathrm{~m} / \mathrm{s}$ ) and the influence of the upper cover. For comparison a grid is included in these figures, the distance between lines is $10 \mathrm{~m}$, except the first horizontal line located at $8 \mathrm{~m}$ above the ground. As expected the upper cover deflects horizontally the plume, and enhances the dispersion in the streamwise direction, producing slightly higher natural gas concentrations in that direction. Therefore, according to the simulations carried out with FLUENT, it is clear that the upper cover has an obvious influence in the dispersion pattern of the gas cloud. The local disturbances produced by the upper lid can be seen in Fig. 4 where it is shown in the vertical symmetry plane the velocity vectors and the mass concentration of natural gas. The upper cover makes the cloud disperse almost parallel to the ground, being this effect more accentuated in case of high wind velocity, making less

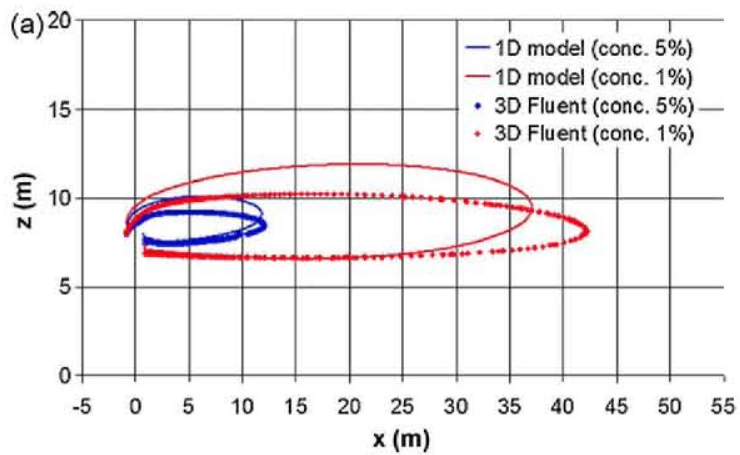

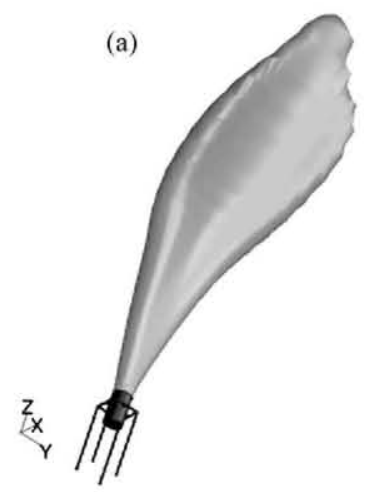

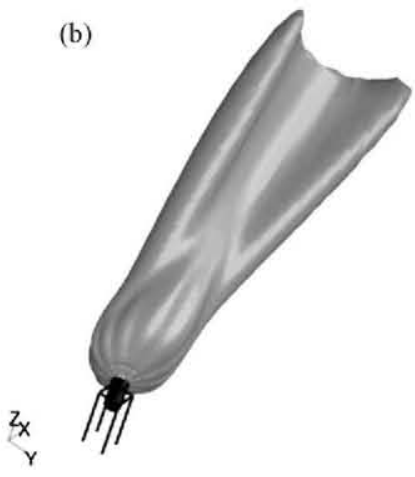

Fig. 10. Three-dimensional view of the cloud corresponding to a concentration equal to $5 \%$. Mass flow rate is $70 \mathrm{~kg} / \mathrm{s}$ and the wind velocity is $v=2 \mathrm{~m} / \mathrm{s}$ : (a) without upper cover and (b) with upper cover.
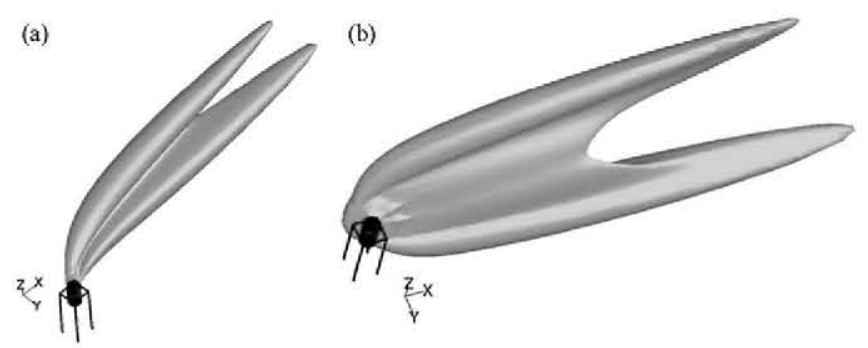

Fig. 11. Three-dimensional view of the cloud corresponding to a concentration equal to $5 \%$. Mass flow rate is $70 \mathrm{~kg} / \mathrm{s}$ and the wind velocity is $v=10 \mathrm{~m} / \mathrm{s}$ : (a) without upper cover and (b) with upper cover.

apparent the buoyancy properties of natural gas. This simulation confirmed the detected presence of low concentrations of natural gas outside the exclusion zone in the compressor station.

In Figs. 10 and 11, three-dimensional views of the plume are given, both with and without upper lid and the same mass flow rate, but each figure with a different wind velocity. As indicated by List the cross-flow appears to see the jet as a solid object occurring vortex shedding, that induces stream-wise vorticity which may become sufficiently strong to bifurcate the flow, see Scorer Turner Crabbet al. Diez et al. According to Abdelwahed and Chow buoyancy within the jet exacerbates the splitting. According to our results, the twolobule structure is more apparent for the high wind velocity, and the tendency for bifurcation is higher when there is the upper

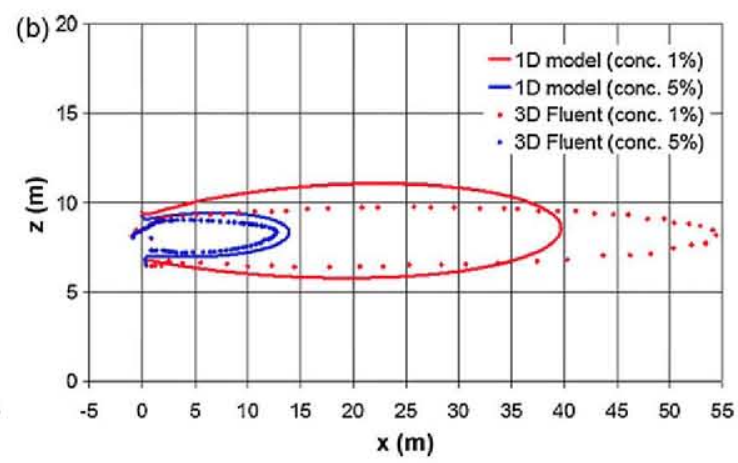

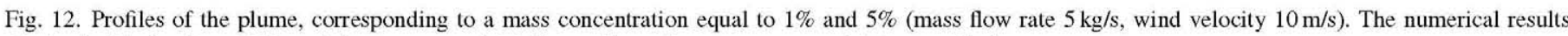
obtained with the 1D and 3D model are compared. (a) Case without the upper cover and (b) case with the upper cover. 

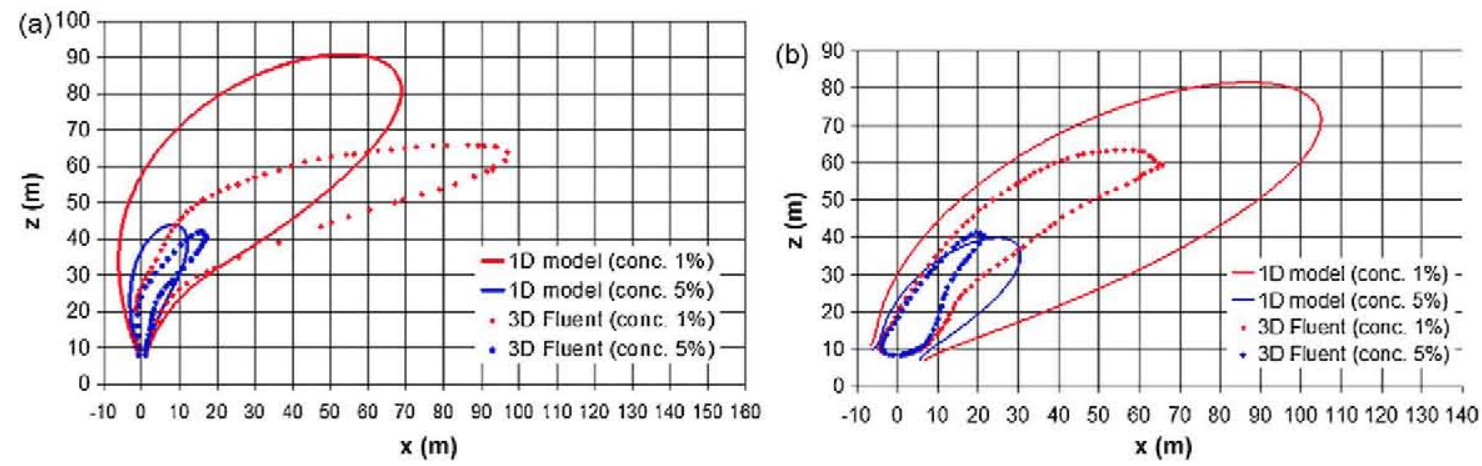

Fig. 13. Profiles of the plume, corresponding to a mass concentration equal to $1 \%$ and $5 \%$ (mass flow rate $70 \mathrm{~kg} / \mathrm{s}$, wind velocity $2 \mathrm{~m} / \mathrm{s}$ ). The numerical results obtained with the 1D and 3D model are compared. (a) Case without the upper cover and (b) case with the upper cover.
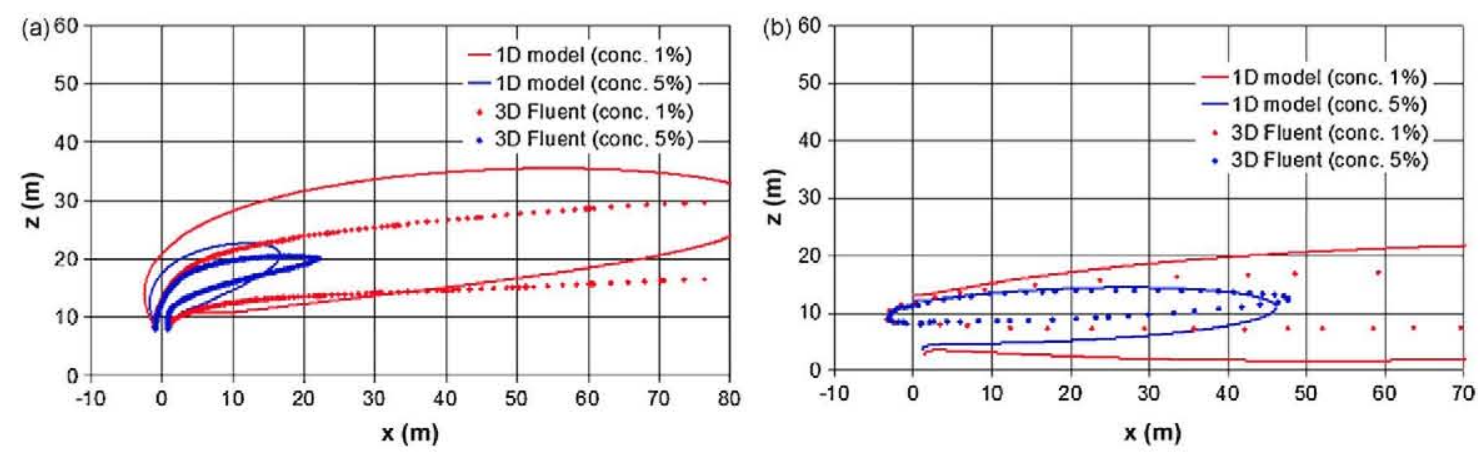

Fig. 14. Profiles of the plume, corresponding to a mass concentration equal to $1 \%$ and $5 \%$ (mass flow rate $70 \mathrm{~kg} / \mathrm{s}$, wind velocity $10 \mathrm{~m} / \mathrm{s}$ ). The numerical results obtained with the 1D and 3D model are compared. (a) Case without the upper cover and (b) case with the upper cover.

lid, as can be seen in Figs. 10 and 11. In our case, the natural gas is released trough two concentric rings, but in order to check the influence of the exit geometry on the shape of the cloud, simulations with a unique equivalent exit surface are carried out and a similar shape of the cloud is obtained.

\subsection{Plume trajectories and comparison of results obtained with the two models}

In Figs. 12-14, the iso-concentration lines, for $1 \%$ and $5 \%$, calculated with the one-dimensional and three-dimensional models are compared. As it is obvious the $1 \mathrm{D}$ model can never reproduce the three-dimensional aspects, such as the two-lobule structure or the different diffusion in vertical and horizontal directions. However, taking into account these limitations the agreement is reasonably good. The results represented correspond to the centre line value in the one-dimensional model.

Some of the discrepancies appearing in these Figs. 12-14 may be explained because the distribution of natural gas concentration is seldom similar to a Gaussian distribution (see Figs. 15-17). In particular, the centre line value used in the 1D model may not coincide with the centre value calculated with the 3D model, see for example, the case where there is obviously bifurcation, presented in Fig. 17b. In the following sections,

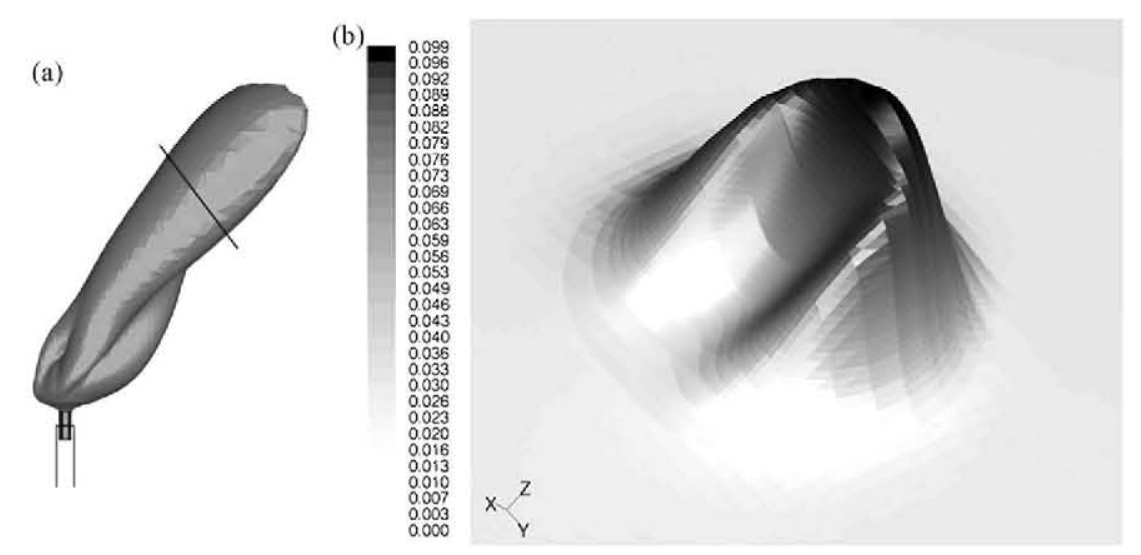

Fig. 15. (a) Three-dimensional view of the cloud corresponding to a concentration equal to $5 \%$. A black line indicates the section used for the mass fraction distribution showed in (b). Mass flow rate is $70 \mathrm{~kg} / \mathrm{s}$ and the wind velocity is $v=2 \mathrm{~m} / \mathrm{s}$. With upper cover. 

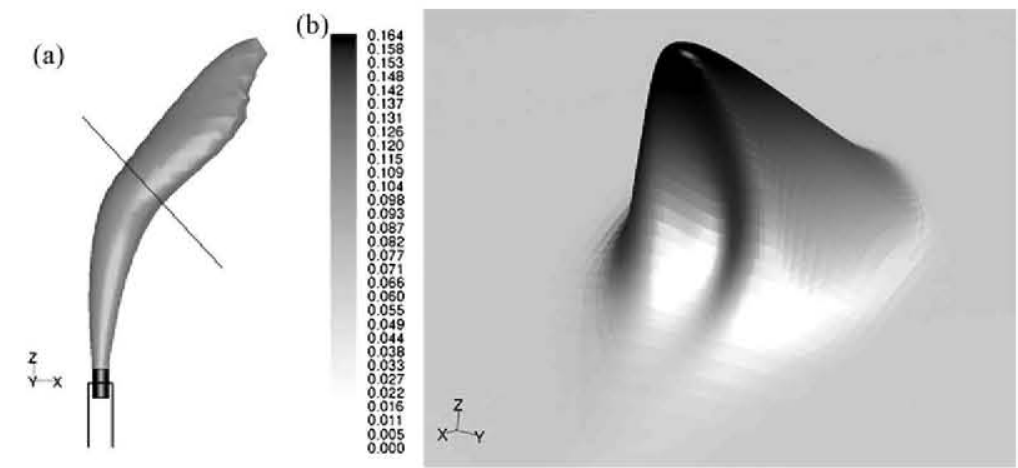

Fig. 16. (a) Three-dimensional view of the cloud corresponding to a concentration equal to $5 \%$. A black line indicates the section used for the mass fraction distribution showed in (b). Mass flow rate is $70 \mathrm{~kg} / \mathrm{s}$ and the wind velocity is $v=2 \mathrm{~m} / \mathrm{s}$. Without upper cover.

when comparing the $1 \mathrm{D}$ and $3 \mathrm{D}$ results, the centre line values will be used for the 1D model and the maximum value of the concentration of the cross section for the 3D results. In Fig. 13a it can be observed that the $1 \%$ contour calculated with the $1 \mathrm{D}$ model is more vertical than the one obtained with the 3D model; this may be explained because the wind velocity considered in the 1D model is uniform, equal to the one corresponding to the exit whereas, in the 3D model the wind velocity increases with height.

\section{Non-dimensional representation of the results}

In cases in which the initial mass flux is low enough so that the following condition is satisfied:

$\mu_{1}=\frac{m_{0} F_{0}^{1 / 2}}{\pi \alpha^{1 / 2} M_{0}^{5 / 4}} \ll 1$

(where $\alpha, m_{0}, M_{0}$ and $F_{0}$ have introduced in Section 2) it can be assumed that there is no mass flux, but there is a net momentum flux, $M_{0}$, at the exit, so that Eqs. (8) and (9) become:

$s=0 \rightarrow \pi b^{2} u=0$

$s=0 \rightarrow \pi b^{2} u^{2}=M_{0}$

This means that for typical distances downstream of the exit the mass entrained is much larger than the actual mass flow rate at the exit. Condition (14) is not always satisfied, and then these boundary conditions are applied at a virtual origin, which does not coincide with the real exit. For cases 2, 3 and 7 of Table 1, without the upper cover the above condition is satisfied. For cases with upper cover, in which, as indicated in Section 2, most of the initial momentum is taken away by the lid, $M_{0}$ will be very small and condition ( $\left.8^{\prime}\right)$ will not be necessarily satisfied; a separate analysis will be made in the next subsection for the cases in which there is an upper cover.

If condition (14) is satisfied the following change of variables suggested by Morton [23] (see Turner [24]) for vertical plumes, can reduce considerably the number of parameters appearing in the solution of the previous system of equations:

$$
\begin{aligned}
& s=\alpha^{-1 / 2} M_{0}^{3 / 4} F_{0}^{-1 / 2} s_{1} \\
& b=\alpha^{1 / 2} M_{0}^{3 / 4} F_{0}^{-1 / 2} b_{1} \\
& u=\alpha^{-1 / 2} M_{0}^{-1 / 4} F_{0}^{1 / 2} u_{1} \\
& U=\alpha^{-1 / 2} M_{0}^{-1 / 4} F_{0}^{1 / 2} U_{1} \\
& g \frac{\rho_{\mathrm{a}}-\rho}{\rho_{\mathrm{a}}}=\alpha^{-1 / 2} M_{0}^{-5 / 4} F_{0}^{3 / 2} Y_{\mathrm{N} 1}
\end{aligned}
$$

where, $s_{1}, b_{1}, u_{1}, U_{1}, Y_{\mathrm{N} 1}$ are the non-dimensional plume coordinate, the plume radius, the plume velocity, the wind velocity and the natural gas concentration, respectively. If these new variables are introduced in the system of Eqs. (4)-(7), and the simplified boundary conditions $\left(8^{\prime}\right)$ and $\left(9^{\prime}\right)$ are used, the solution of the system of equations turns to depend only on the non-dimensional wind velocity $U_{1}$, and the ratio of entrainment coefficients $\beta / \alpha$
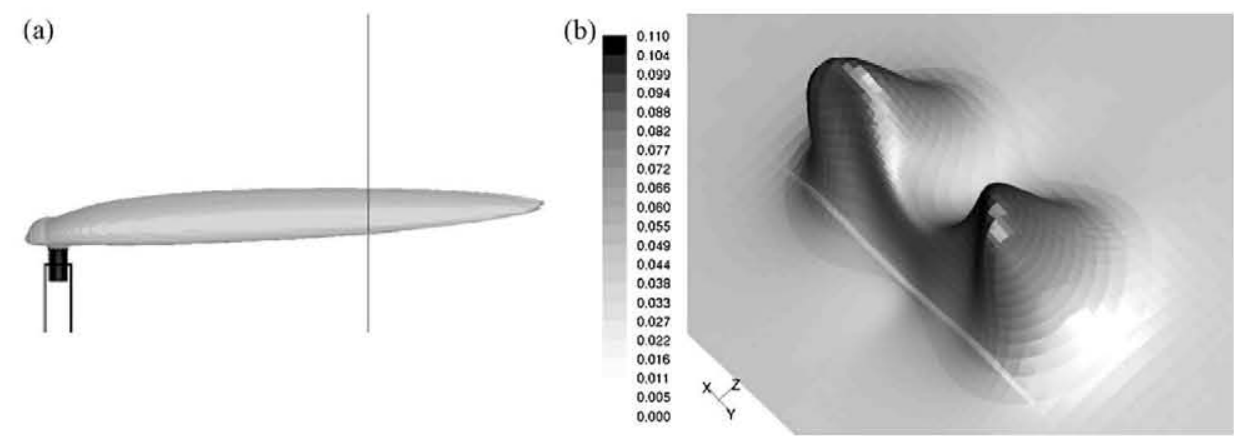

Fig. 17. (a) Three-dimensional view of the cloud corresponding to a concentration equal to $5 \%$. A black line indicates the section used for the mass fraction distribution showed in (b). Mass flow rate is $70 \mathrm{~kg} / \mathrm{s}$ and the wind velocity is $v=10 \mathrm{~m} / \mathrm{s}$. With upper cover. 
and $\varepsilon / \alpha$. In particular, the boundary conditions (8) and (9) will become in the new variables:

$s_{1}=0 \rightarrow b_{1}^{2} u_{1}=\mu_{1} \ll 1$,

$s_{1}=0 \rightarrow b_{1}^{2} u_{1}^{2}=\frac{1}{\pi}$,

thus confirming the hypothesis of Eq. (8') if condition (14) is satisfied. In Table 1 the values of $\mu_{1}$ are given.

Using the non-dimensional variables previously defined, we can reduce the problem of the silencer without the upper lid with different mass flow rates and wind velocities to one depending only of the non-dimensional wind velocity, $U_{1}$. However, this approach can be only applied to mass flow rates high enough, so that condition (14) is satisfied, and the distance between the exit and the virtual origin is small, otherwise the plume obtained with non-dimensional variables will deflect before the real exit. For the cases studied in this work, only those corresponding to a mass flow rate equal to $70 \mathrm{~kg} / \mathrm{s}$ satisfy this condition.

The non-dimensional plume trajectories are given in Fig. 18, for different values of $U_{1}$, where $x_{1}$ and $z_{1}$ have been made non-dimensional as $s_{1}$ in Eq. (15). As expected the trajectories become more inclined as the wind velocity increases. The agreement between one-dimensional model and three-dimensional results is quite good for the cases 3 and 7 that satisfy the condition (14). Case $5\left(\mu_{1}=0.23\right)$ has been included in the figure; however the agreement is not good because condition (14) is not satisfied. In case 2, the trajectory calculated with the $1 \mathrm{D}$ model is more vertical than the one obtained with the 3D model; as in Fig. 13a this may be explained because the wind velocity considered in the 1D model is the one corresponding to the exit whereas, in the 3D model the wind velocity increases with height.

The non-dimensional parameter $U_{1}$ may be written as $U_{1}=\alpha^{1 / 2}\left(z_{\mathrm{M}} / z_{\mathrm{B}}\right)^{1 / 2}$, where $z_{\mathrm{M}}=M_{0}^{1 / 2} / U$ is a typical length associated to momentum dominated jets, and $z_{\mathrm{B}}=F_{0} / U^{3}$ is a typical length associated to a buoyant dominated plume. List [10] indicates that when $z_{\mathrm{M}}>z_{\mathrm{B}}$ jet momentum is dominant,

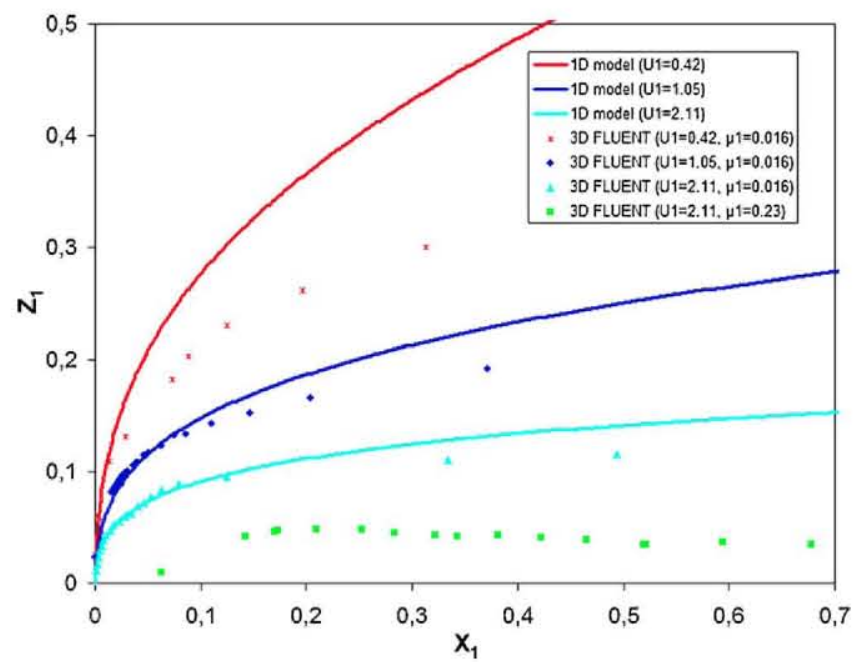

Fig. 18. Non-dimensional centre lines of the plumes for different nondimensional velocities and without the upper cover. and when $z_{\mathrm{M}}<z_{\mathrm{B}}$ jet buoyancy is important. In our case this means that when $U_{1}>\alpha^{1 / 2}=0.35$ momentum dominates, and when $U_{1}<\alpha^{-1 / 2}=0.35$ buoyancy is more important. So, all the cases studied may be considered as momentum dominated. For this situation List [10] proposes a correlation $z / z_{\mathrm{M}}=C_{2}\left(x / z_{\mathrm{M}}\right)^{1 / 3}$ valid for moderately large values of $z / z_{\mathrm{M}}$, and $z / z_{\mathrm{B}}=C_{4}\left(x / z_{\mathrm{B}}\right)^{2 / 3}$ valid for very large values of $z / z_{\mathrm{M}}$. Using the change of variable given in Eqs. (15) and (18), these correlations may be expressed as:

$z_{1}=C_{2}\left(\frac{\alpha}{U_{1}}\right)^{2 / 3} x_{1}^{1 / 3}, \quad$ for $\quad x_{1} \ll 1$

$z_{1}=C_{4} \frac{\alpha^{2 / 3}}{U_{1}} x_{1}^{2 / 3}$, for $x_{1} \approx 1$

The trajectories indicated in Fig. 18 correspond approximately with these correlations taking $C_{2} \approx 1.3$ and $C_{4} \approx 1.3$, $C_{2}$ is slightly lower than that indicated in List but $C_{4}$ if of the same order of that proposed by List.

In Fig. 19 is presented the evolution of the non-dimensional natural gas concentration with non-dimensional coordinate, $s_{1}$, for different values of $U_{1}$, it can be observed, as expected, that the concentration decreases with $U_{1}$. In this figure, are also presented for comparison results from the 3D analysis, the agreement is good. The values calculated with the 3D model correspond to the maximum ones in each cross-section, and those obtained with the 1D model are those at the centre. Besides, the classical result for the evolution of mass concentration in vertical plumes, when $U=0$, has been added to the figure. This limit result can be obtained from the following solution of Eqs. (4)-(7): $b=A \alpha s, u=B\left(F_{0} / \alpha^{2}\right)^{1 / 3}\left(1 / S^{1 / 3}\right)$, $Y_{\mathrm{N}}=C\left[\rho_{\mathrm{a}} /\left(\rho_{\mathrm{a}}-\rho_{\mathrm{N}}\right)\right](1 / g)\left(F_{0}{ }^{2 / 3} / \alpha^{4 / 3} s^{5 / 3}\right)$, where $A=6 / 5$, $B=[75 /(144 \pi)]^{1 / 3}=0.55$, and $C=1 /\left(A^{2} B \pi\right)=0.40$. In the nondimensional variables these results can be expressed as $b_{1}=A s_{1}$, $u_{1}=B\left(1 / s_{1}^{1 / 3}\right), \quad Y_{\mathrm{N} 1}=C\left(1 / s_{1}^{5 / 3}\right)$. The value presented in Fig. 18 is the centre value, that according to Eq. (2) should be twice the average one: $Y_{\mathrm{Ncl}}=2 C\left(1 / s^{5 / 3}\right)$. This result coin-

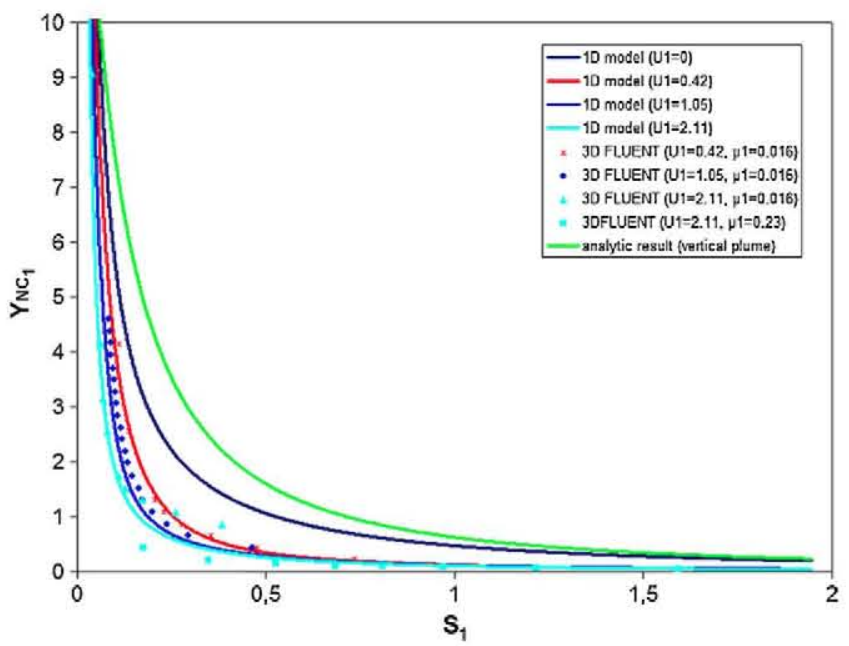

Fig. 19. Evolution of the non-dimensional mass concentration of natural gas, for different velocities and without the upper cover. The 1D results are centre line values and the $3 \mathrm{D}$ results maximum values. 
cides with the one obtained from the $1 \mathrm{D}$ model for $U_{1}=0$ given in Fig. 19, only for large values of $s_{1}$, this is due to the fact that this analytical solution does not satisfy the initial condition (9"). If the origin of $s_{1}$ is slightly displaced the following correlation is obtained that fits approximately the $1 \mathrm{D}$ model for $U_{1}=0$ :

$Y_{\mathrm{Nc} 1}=2 C \frac{1}{\left(s_{1}+0.16\right)^{5 / 3}}$

This analytical result can be interpreted as an upper limit and may be useful for safety purposes.

\subsection{Simulation of cases with upper cover}

From Eq. (5) it can be seen that the momentum flux increases with $s$, because of the buoyancy term, so that there can be situations in which the total momentum is much larger than its initial value, $M_{0}$, within short distances from the exit. This has been found to be a good approximation for cases in which there is the upper cover and consequently the values of $M_{0}$ are small. We are going to consider cases in which boundary conditions (8) and (9) will be the following:

$s=0 \rightarrow \pi b^{2} u=m_{0}$

$s=0 \rightarrow \pi b^{2} u^{2}=0$

If the initial momentum flux is low enough because of the presence of the cover, the boundary conditions ( 8 "') and (9"') will have to be used, and the initial momentum $M_{0}$ will not appear. Therefore, eliminating $M_{0}$ from the previous non-dimensional variables and using instead the wind velocity $U$, the following new non-dimensional variables are obtained:

$s=\frac{F_{0}}{\alpha^{2} U^{3}} s_{2}$

$b=\frac{F_{0}}{\alpha U^{3}} b_{2}$

$u=U u_{2}$

$Y_{\mathrm{N}}=\frac{\rho_{\mathrm{a}} \alpha^{2} U^{5}}{g\left(\rho_{\mathrm{a}}-\rho_{\mathrm{N}}\right) F_{0}} Y_{\mathrm{N} 2}$

Now the wind velocity has been used to make the variables non-dimensional, and all the independent plume variables, depend only on the non-dimensional coordinate, $s_{2}$, and the nondimensional mass flux $\mu_{2}$. Using this change of variables in Eqs. (8) and (9), the initial values of the non-dimensional mass and momentum fluxes will be:

$s_{2}=0 \rightarrow b_{2}^{2} u_{2}=\mu_{2}=\frac{m_{0} \alpha^{2} U^{5}}{\pi F_{0}^{2}}$,

$s_{2}=0 \rightarrow b_{2}^{2} u_{2}^{2}=\Lambda_{2}=\frac{M_{0} \alpha^{2} U^{4}}{\pi F_{0}^{2}} \ll 1$

Condition (9'"') $\Lambda_{2} \ll 1$ can always be satisfied if the lid takes out enough momentum from the outgoing jet. In Table 1

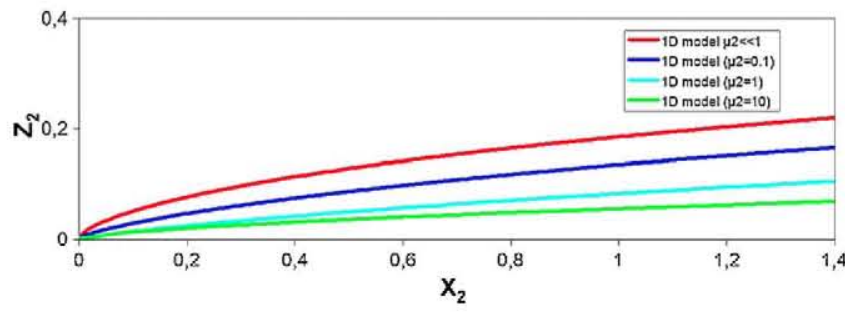

Fig. 20. Non-dimensional centre lines of the plumes for different values of $\mu_{2}$ obtained with the one-dimensional model for cases with upper cover.

the values of $\mu_{2}$ and $\Lambda_{2}$ are given. Cases 1, 4 and 6 from Table 1 satisfy this condition. If $\mu_{2}$ is of order one or much smaller than one, the condition ( $\left.9^{\prime \prime \prime}\right)$ will be satisfied if:

$\frac{\Lambda_{2}}{\mu_{2}}=\frac{u_{0}}{U} \ll 1$,

so that the effect of the lid, decreasing $u_{0}$, will be reinforced in high winds.

In Fig. 20 the non-dimensional plume trajectory, obtained with the one-dimensional model, is given for different values of parameter $\mu_{2}$, where again, $x_{2}$ and $z_{2}$ have been made nondimensional as $s_{2}$ in Eq. (23). As it can be seen in the figure, when the parameter $\mu_{2}$ increases (high wind velocity and low buoyancy) the plume tends to disperse horizontally. It can observed that, using the buoyancy length $z_{\mathrm{B}}=F_{0} / U^{3}$ in Eq. (23), the following relationship holds $s_{2}=\left(s / z_{\mathrm{B}}\right) \alpha^{2}$, then for values of $s_{2}$ of order one $\left(s / z_{\mathrm{B}}\right) \approx \alpha^{-2} \gg 1$, and the flow can be considered as buoyancy dominated, see List [10]. The trajectories indicated in Fig. 19 correspond approximately with the law $z_{2}=C_{\mathrm{B}} x_{2}^{2 / 3}$, the value of $C_{B}$ being of the order of 0.2 for $\mu_{2}=0$. List [10] gives the following correlation, mentioned previously, for buoyancy dominated flows, $z / z_{\mathrm{B}}=C_{4}\left(x / z_{\mathrm{B}}\right)^{2 / 3}$, then, the following relationship holds between the two coefficients $C_{\mathrm{B}}=C_{4} \alpha^{2 / 3}$, yielding a value of $C_{4} \approx 0.8$ for $\mu_{2}=0$, that is within the range of the values quoted by List. For larger values of $\mu_{2}$, this coefficient becomes smaller and for $\mu_{2}=10, C_{4} \approx 0.25$, much smaller than the values cited by List [10]. As can be seen in Fig. 20, as $\mu_{2}$ increases the trajectories become more horizontal; this can be explained because $\mu_{2}$ (Eq. ( $8^{\prime \prime \prime \prime)) ~ i n c r e a s e s ~ w i t h ~ t h e ~ w i n d ~ v e l o c i t y, ~ a n d ~}$ decreases with the buoyancy and both factors should tend to make the trajectories more horizontal. For large values of $x_{2}$ all the trajectories tend to become independent of $\mu_{2}$.

A comparison between the one-dimensional model and threedimensional trajectories is presented in Fig. 21 (cases 4 and 6) and Fig. 22 (case 1). The agreement is reasonably good for the lower values of $\mu_{2}$, however, for the case $4\left(\mu_{2}=9.39\right)$ the non-

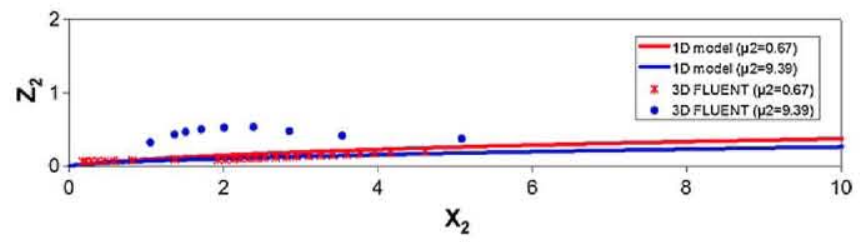

Fig. 21. Comparison of the non-dimensional centre lines of the plumes, for cases 4 and 6 , obtained with the one-dimensional model and the three-dimensional code. 


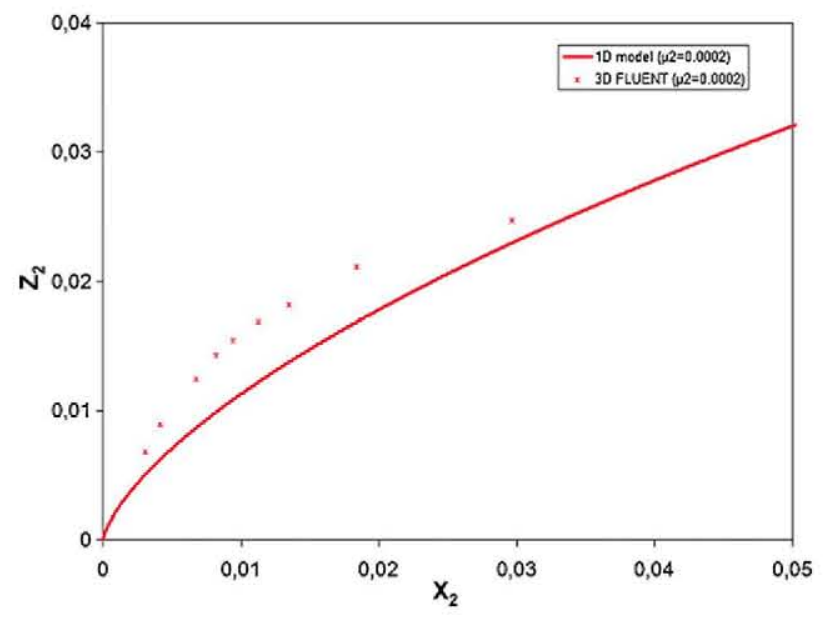

Fig. 22. Comparison of the non-dimensional centre line of the plume, for case 1 , obtained with the one-dimensional model and the three-dimensional code.

dimensional treatment fails at the initial stages, but far enough the tendency seems to be correct.

The variation of the non-dimensional concentration, $Y_{\mathrm{Nc} 2}$, with non-dimensional downstream distance, $s_{2}$, is represented in Fig. 23 for different values of $\mu_{2}$. A good correlation for the variation of concentration for $\mu_{2} \ll 1$ is:

$Y_{\mathrm{Nc} 2}=\frac{0.2}{s_{2}^{5 / 3}}$,

that is undistinguishable from the curve of Fig. 23. In both Figs. 24 and 25, are also presented for comparison results from the $3 \mathrm{D}$ analysis, the agreement is quite good for cases $1\left(\mu_{2}=0.0002\right)$ and $4\left(\mu_{2}=9.39\right)$, but for case $6\left(\mu_{2}=0.67\right)$, the agreement is good only for distances large enough. In all cases the tendencies predicted by the two calculation procedures are in agreement.

As in the previous section, the values calculated with the $3 \mathrm{D}$ model correspond to the maximum ones in each cross-section, and those obtained with the 1D model are those at the centre.

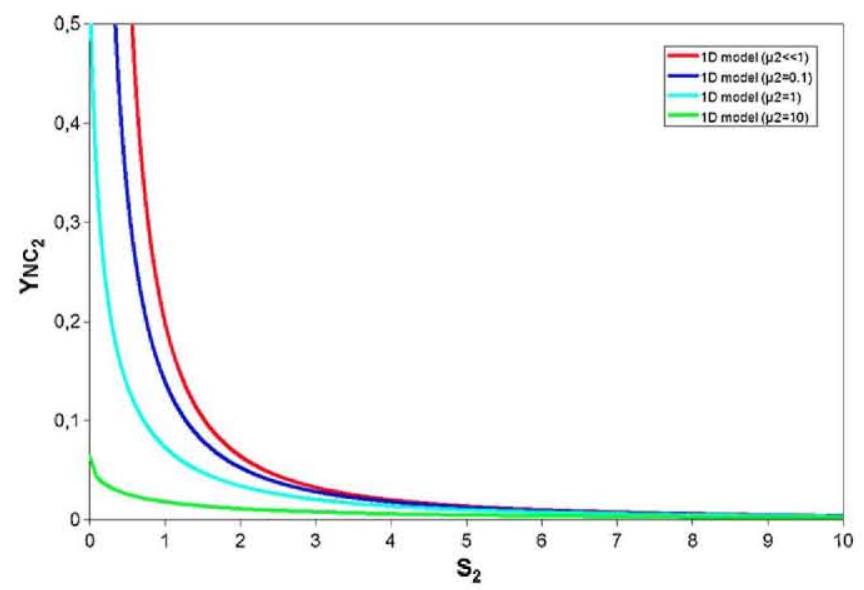

Fig. 23. Evolution of the mass concentration along the centre line for different values of $\mu_{2}$ obtained with the one-dimensional model for cases with upper cover. The $1 \mathrm{D}$ results are centre line values and the $3 \mathrm{D}$ results maximum values.

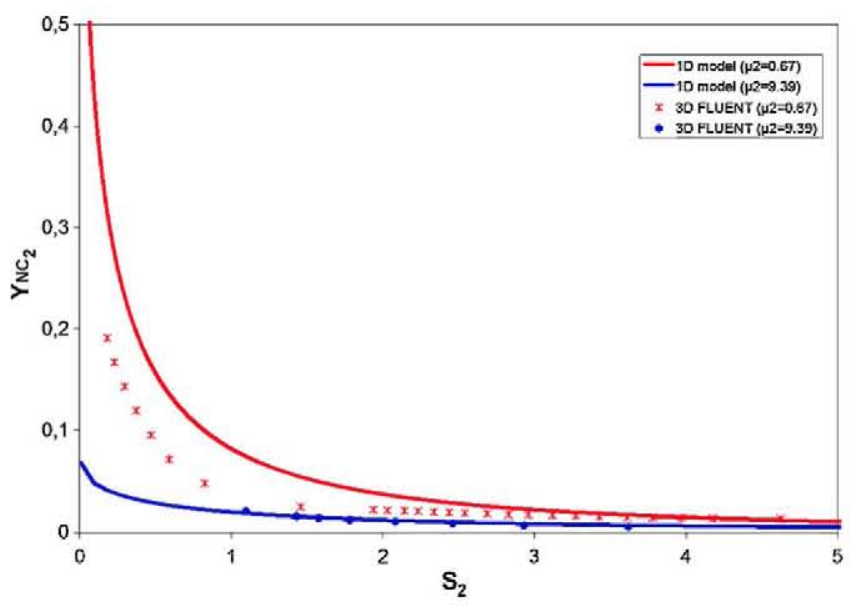

Fig. 24. Comparison of the non-dimensional centre line of the plume, for cases 4 and 6 , obtained with the one-dimensional model and the three-dimensional code. The $1 \mathrm{D}$ results are centre line values and the $3 \mathrm{D}$ results maximum values.

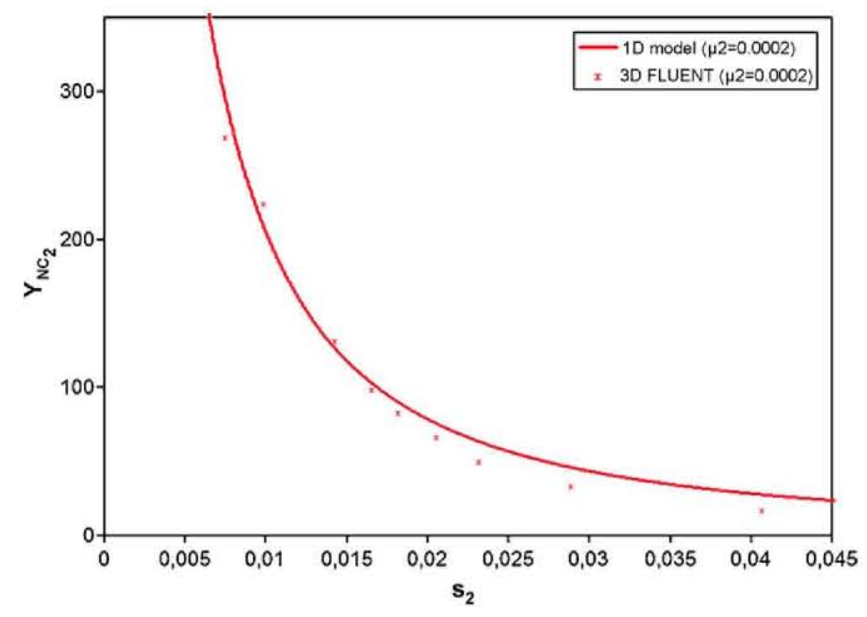

Fig. 25. Comparison of the non-dimensional centre line of the plume, for case 1 , obtained with the one-dimensional model and the three-dimensional code.

\section{Conclusions}

According to the simulations carried out with the threedimensional model, it is clear that the upper cover has an obvious influence in the dispersion pattern of the gas cloud. The upper cover makes the cloud disperse almost parallel to the ground, being this effect more accentuated in case of high wind velocity, canceling the buoyancy properties of natural gas. This simulation confirmed the detected presence of low concentrations of natural gas outside the exclusion zone in the compressor station. Considering our results, the two-lobule structure is more apparent for the high wind velocity, and the tendency for bifurcation is higher when there is the upper lid.

A one-dimensional model is proposed that reproduces reasonably well the behavior and tendencies of the plume dispersion. To compare the $1 \mathrm{D}$ and $3 \mathrm{D}$ results non-dimensional variables have been introduced. For the cases without the upper cover the variables have been made non-dimensional with the initial momentum flux and the buoyancy flux, then, the nondimensional wind velocity is considered as a parameter, and the non-dimensional mass flux is considered as a very small 
quantity. On the other hand, for the cases with the upper cover the variables have been made non-dimensional with the wind velocity and the buoyancy flux, then, the non-dimensional initial mass flux is considered as a parameter, and the non-dimensional initial momentum flux is considered as a very small quantity. This assumption permits to take into account the effect of the upper cover in the diffusion of the plume in the 1D model. This condition $\Lambda_{2} \ll 1$ is satisfied if the lid takes from the outgoing jet enough momentum, so that, the resulting velocity is much smaller than the wind velocity.

For both cases with and without the upper cover, and low enough values of the initial mass flux, $\mu_{2} \ll 1$ and $\mu_{1} \ll 1$, respectively, the one-dimensional model predicts reasonably well the natural gas concentration. However, for the cases with the upper lid and moderate values of the non-dimensional initial mass flux, $\mu_{2}$, the gas concentration values obtained with the one-dimensional model are larger than those calculated with the FLUENT code. The behavior of the plume trajectory is well reproduced downstream, but near the exit there are discrepancies for some cases when there is an upper lid, this may be due to the three-dimensional interactions between the silencer and plume that cannot be reproduced by the one-dimensional model.

\section{Acknowledgment}

The data and support provided by Department of Technology of Enagas S.A. are gratefully acknowledged by the authors.

\section{References}

Lees' Loss Prevention in the Process Industries, Hazard Identification, Assessment and Control, Elsevier Butterworth-Heinemann, third ed., 2005. J.M. Santamaría, P.A. Braña, Análisis y Reducción de Riesgos en la Industria Química, Editorial Mapfre, la Edición, 1994.

S.Y. Liao, Q. Cheng, D.M. Jiang, J. Gao, Experimental study of flammability limits of natural gas-air mixture, J. Hazard. Mater. 119 (1-3) (2005) $81-84$

S.Y.Liao, D.M. Jiang, Z.H. Huang, Q. Cheng, J. Gao, Y. Hu, Approximation of flammability region for natural gas-air-diluent mixture, J. Hazard. Mater. 125 (1-3) (2005) 23-28.

European Standard, EN61779-1:2002 Electrical apparatus for the detection and measurement of flammable gases, Part 1: General requirement and test methods.
F. Castro, J. Servert, F. Manuel, C. Lopez Piñon, Crespo, A. Modelo numérico de descargas de gas natural, Procceding IX Congreso Nacional de Ingeniería Química, Zaragoza, Spain, 1990.

J. Servert, A. Crespo, J. Hernández, One-dimensional model of a turbulent jet diffusion flame in an ambient atmospheric flow, derived from a three-dimensional model, Combust. Sci. Technol. 124 (1997) 83114.

G.A. Briggs, Discussion of a comparison of the trajectories of rising buoyant plumes with theoretical empirical models, Atmos. Environ. 9 (1975) 455-462.

G. Ooms, A.P. Mahieu, A comparison between a plume path model and a virtual point source model for a stack plume, Appl. Sci. Res. 36 (1981) 339-356.

E.J. List, Turbulent jets and plumes, Annu. Rev. Fluid Mech. 14 (1982) 189-213.

D. Contini, A. Robins, Water tank measurements of buoyant plume rise and structure in cross flow, Atmos. Environ. 35 (2001) 61056115.

R.W. MacDonald, R.K. Strom, P.R. Slawson, Water flume study of the enhancement of buoyant rise in pairs of merging plumes, Atmos. Environ. 36 (2002) 4603-4615.

A. Seifert, L. Shemer, Plume rise from a chimney with an elongated exit cross sections, Atmos. Environ. 29 (1995) 709-713.

M.P. Escudier, Aerodynamics of a burning turbulent gas jet in a crossflow, Combust. Sci. Technol. 4 (1972) 293-301.

F. Pasquill, F.B. Smith, Atmospheric Diffusion, Ellis Horwood Ltd., 1983. E. Gallego, E. Migoya, J.M. Martín-Valdepeñas, A. Crespo, J. García, A. Venetsanos, E. Papanikolau, S. Kumar, E. Studer, O.R. Hansen, Y. Dagba, T. Jordan, W. Jahn, S. Hoiset, D. Makarov, J. Piechna, An intercomparison exercise on the capabilities of CFD models to predict distribution and mixing of $\mathrm{H} 2$ in a closed vessel, Int. J. Hydrogen Energy 32 (2007) 2235-2245

E. Migoya, A. Crespo, J. García, F. Moreno, F. Manuel, A. Jiménez, A. Costa, Comparative study of the behavior of wind-turbines in a wind farm, Energy 32 (2007) 1871-1885.

R.S. Scorer, The behaviour of chimney plumes, Int. J. Air Pollut. 1 (1959) 198-220.

J.S. Turner, A comparison between vortex ring and vortex pairs, J. Fluid Mech. 1 (1960) 419-432.

D. Crabb, D.F.G. Durao, J.H. Whitelaw, A round jet normal to a cross-flow, Trans. ASME J. Fluids Eng. 103 (1981) 142-153.

F.J. Diez, L.P. Bernal, G.M. Faeth, PLIF and PIV measurements of the selfpreserving structure of steady round buoyant turbulent plumes in crossflow, Int. J. Heat Fluid Flow 26 (2005) 873-882.

M.S.T. Abdelhwahed, V.H. Chow, Bifurcation of buoyant jets in cross flow, Tech. Rep. 78-1, Dept. Civ. Eng. McGill Univ., 1978.

B.R. Morton, Forced plumes, J. Fluid Mech. 5 (1959) 173-200.

J.S. Turner, Buoyancy Effects in Fluids, Cambridge University Press, 1979. 